\title{
345. Histochemische Untersuchungen von intestinalen Metaplasmen der Magenschleimhaut beim Magencarcinom
}

\author{
G. H. Rühl ${ }^{1}$, W. Kozuschek ${ }^{2}$, R. W. Veh ${ }^{3}$ und W. Hartung ${ }^{1}$ \\ ${ }^{1}$ Institut für Pathologie der Ruhr-Universität Bochum, ${ }^{2}$ Chirurgische Universitätsklinik Bochum, \\ Knappschaftskrankenhaus, ${ }^{3}$ Institut für Anatomie der Ruhr-Universität Bochum, D-4630 Bochum
}

\section{Histochemical Study on Intestinal Metaplasia of Gastric Mucosa in Cancerous Stomachs}

Summary. Intestinal metaplasia (IM) can be divided morphologically and histochemically into a 'jejunal' type and 'colonic' type, as well as a complete and incomplete type. The premalignant potential of the subtypes, however, remains controversial. Individually examined IM lesions in 30 stomachs resected for ulcer and carcinoma showed an exceptionally frequent occurrence of sulphomucins in the carcinoma group. A histochemically colon-like IM with detection of sulphomucins and O-acetylated sialomucins was not found to be more frequent in the carcinoma group.

Key words: Carbohydrate histochemistry - O-acetylated sialic acids - Intestinal metaplasia - Gastric carcinoma.

Zusammenfassung. Die intestinale Metaplasie (IM) kann an Hand histochemischer und morphologischer Kriterien in einen Jejunumtyp und in einen Colontyp einerseits sowie in einen kompletten Typ und in einen inkompletten Typ andererseits unterschieden werden. Der prämaligne Charakter der Subtypen bleibt jedoch umstritten. Einzeln ausgezählte IM-Herde in 30 Ulcus- und Carcinommägen zeigen ein überdurchschnittlich häufiges Auftreten von Sulphomucinen in der Carcinomgruppe. Eine histochemisch colonähnliche IM mit Nachweis von Sulphomucinen und substituierten Sialomucinen war dagegen in der Carcinomgruppe nicht häufiger nachweisbar.

Schlïsselwörter: Carbohydrat-Histochemie - O-acetylierte Sialinsäuren - Intestinale Metaplasie - Magencarcinom.

\section{Akne conglobata: Taktik der chirurgischen Therapie}

\author{
W. Priesack, D. Maroske und H. Hamelmann
}

Chirurgische Universitäts Klinik Kiel, Abteilung Allgemeinchirurgie, D-2300 Kiel, Zentrum für Operative Medizin I, Klinikum der Philipps-Universität, Chirurgische Klinik D-3550 Marburg/Lahn

\section{Acne conglobata - Hidradenitis Suppurativa. A Method of Surgical Therapy}

Summary. Purulent abscess formation of the skin and subcutis signifies the mostly plurilocular clinical pict ure. The definitive therapy has to be surgical, but local antiseptic pre-treatment must precede and antibiotics should only be used in sepsis and for peri-operative prophylaxis. Small lesions should be excised and primarily closed. Large areas require treatment in several stages. Stage $1:$ radical excision of skin and subcutis involved, including the fistulae; stage 2: delayed closure by skin graft, skin flap or musculocutaneous flap; stage 3: flap for correction of scar tissue contraction.

Key words: Acne conglobata - Hidradenitis suppurativa - Surgical therapy.

Zusammenfassung. Multiloculäres Krankheitsbild mit eitrig abscedierenden Herden der Haut und Subcutis. Die definitive chirurgische Therapie erfolgt nach lokal antiseptischer Vorbehandlung. Antibiotica sind bei Sepsis und zur perioperativen Prophylaxe sinnvoll. Kleine Herde werden excidiert und primär verschlossen. Mehrzeitiges Vorgehen bei großflächigen Arealen. 1. Sitzung: Radikale Excision der betroffenen Haut und Subcutis unter Mitnahme des Fistelsystems. 2. Sitzung: Defektdeckung durch Spalthaut, cutane oder musculocutane Lappen. 3. Sitzung: Lappenplastiken zur Narbenkorrektur.

Schliisselwörter: Akne conglobata - Chirurgische Therapie. 